\title{
Relationship between Spiritual Health and Quality of Life in Patients with Cancer
}

\author{
Rafat Mohebbifar ${ }^{1}$, Amir H Pakpour ${ }^{2}$, Azin Nahvijou ${ }^{3}$, Atefeh Sadeghi' ${ }^{2,4}$
}

\begin{abstract}
As the essence of health in humans, spiritual health is a fundamental concept for discussing chronic diseases such as cancer and a major approach for improving quality of life in patients is through creating meaningfulness and purpose. The present descriptive analytical study was conducted to assess the relationship between spiritual health and quality of life in $\mathbf{2 1 0}$ patients with cancer admitted to the Cancer Institute of Iran, selected through convenience sampling in 2014. Data were collected using Spiritual Health Questionnaire and the European Organization for Research and Treatment of Cancer Quality of Life Questionnaire (EORTC-QLQ). Patients' performance was assessed through the Karnofsky Performance Status Indicator and their cognitive status through the Mini-Mental State Examination (MMSE). Data were analyzed in SPSS-16 using descriptive statistics and stepwise linear regression. The results obtained reported the mean and standard deviation of the patients' spiritual health scoreas 78.4 \pm 16 .1 and the mean and standard deviation of their quality of life score as $58.1 \pm 18.7$. The stepwise linear regression analysis confirmed a positive and significant relationship between spiritual health and quality of life in patients with cancer $(\beta=0.688$ and $r=0.00)$. The results of the study show that spiritual health should be more emphasized and reinforced as a factor involved in improving quality of life in patients with cancer. Designing care therapies and spiritual interventions is a priority in the treatment of these patients.
\end{abstract}

Keywords: Spiritual health - quality of life - cancer - Iran

Asian Pac J Cancer Prev, 16 (16), 7321-7326

\section{Introduction}

Cancer is one of the main problems of health systems in various countries (Siegel et al., 2015) and a main cause of death in developing countries (Torre et al., 2015). As the third leading cause of death in Iran, aftercardiovascular diseases and road accidents, cancer is particularly important (Mousavi et al., 2009). Cancer is considered the principal threat to health (Vrinten et al., 2014) and people are highly shocked and disappointedwhen they getdiagnosed with the disease and begin to fear death (Akyuz et al., 2008) and their perception of life then changes in the attempt to cope with their new conditions (Reb, 2007). Because of its fatality, patients develop greater spiritual needs upon diagnosis with cancer. In other words, the individual begins to suffer spiritual distress and massive spiritual crises, leading to the loss of his self-esteem and faith. His personal relationships become impaired as a result of the uncertainty of his prospects and periods of hospitalization generate a sense of loneliness and a kind of spiritual crisis in him (Rezaei et al., 2008). Creating a sense of spiritual healthas a main component of mental health is an appropriate way for coping with cancer and its tensions (McClain et al., 2003); individuals who possess this facilitating spiritual factor cope more quickly with cancer (Baider et al., 2003). As the fourth dimension of health, spiritual health unifies the physical, mental and social dimensions of being and includes the existential and religious dimensions of life as well. Religious health is the satisfaction derived from connection to an infinite being -to God-, and existential health is the attempt for understanding the meaning and purpose of life and gaining satisfaction from it (Riley et al., 1998). In general, people with life-threatening and chronic diseases are faced with questions about the meaning and purpose of life, and many of them recognize spiritual health as a factor that gives meaning and purpose to life and improves the quality of life (Mauk and Schmidt, 2004). Quality of life has a multidimensional structure that includes physical and mental health as well as social and cognitive functions (Manandhar et al., 2014). According to the definition provided by the World Health Organization, quality of life refers to people's own understanding of their position in life based on the culture and value system in which they live and based on the relationship between this understanding and their goals, expectations, standards and priorities (Bottomley, 2002). In other words, quality of life is the gap between the individual's current position and

${ }^{1}$ Department of Health Management, School of Health, ${ }^{2}$ Social Determinants of Health Research Center (SDH), ${ }^{4}$ Health Management, Qazvin University of Medical Sciences, Qazvin, ${ }^{3}$ Cancer Research Center, Cancer Institute of Iran, Tehran University of Medical Science, Tehran, Iran *For correspondence: atefeh.sadeghi@ymail.com 
the ideal position hecontemplates (Rose and Yates, 2001). The high prevalence of chronic diseases in the modern world has led to a greater emphasis on the concept of quality of life as a yardstick for evaluating the effects of the disease, treatment and care (Polonsky, 2000). Spiritual health is also a coping strategy in relation to the process of evaluating the quality of life (Jahani et al., 2012). Despite the large number of studies conducted in western countries on the quality of life in patients, the National Cancer Institute of the US has stressed the importance of evaluating the disparities in the results obtained by these studies in order to better understand the effect of cancer and its treatment from the perspective of patients (Rustøen et al., 2010). Given the rising trend of cancer cases across the world, including in Iran, and given the extensive effects of cancer on the quality of life in patients dealing with the disease, identifying the factors that help improve quality of life, such as spiritual health, is necessary. The present study was therefore conducted to assess the relationship between spiritual health and quality of life in patients with cancer.

\section{Materials and Methods}

\section{Study design and samples}

The present cross-sectional study was conducted in 2014 to assess the relationship between spiritual health and quality of life in patients with cancer admitted to the Cancer Institute of Iran (the Cancer Research Center) affiliated to Tehran University of Medical Sciences.

The researcher first obtained the approval of the Medical Ethics Committee and permission to collaborate withthe Cancer Research Center of Tehran University of Medical Sciences and made the necessary arrangements to conduct sampling in the patients admitted to the Cancer Institute. The study inclusion criteria consisted ofbeing older than 18, having received a definite diagnosis of cancer by a specialist, being informed of one's own diagnosis, a Karnofsky score above $40 \%$ and written consent for participation in the study. Patients with mental disorders or who were undergoing chemotherapy and also those unwilling to take part were excluded from the study. Patients who had received a definite diagnosis of cancer by a physician and who were admitted to the surgery clinic or were hospitalized in the surgery wards of the Cancer Institute of Iran and had not yet undergone chemotherapy were introduced to the researcher by the department receptionists and the clinic doctors assistants. The patients' willingness to take part in the study and their demographic details, Karnofsky index, cognitive status and knowledge of own disease were assessed through the demographic and clinical questionnaires prior to beginning the study and 210 eligible patients were ultimately selected through convenience sampling. The researcher provided explanations about the study methods and objectives, ensured participants of the confidentiality of their data, and obtained their written consent before proceeding to the interviews and the collection of the data using the questionnaires.
The patients' demographic data included their age, gender, level of education, economic status, marital status and reason for admission to the center.Their clinical data included the type of cancer, the Karnofsky performance status used for grouping patients according to their functional deficiencies and the cognitive status assessed based on the Mini-Mental State Examination (MMSE). The MMSE is the most common tool used across the world to screen patients for cognitive disorders and has been standardized for different cultures and translated into many different languages, including Persian.First time, Its reliability was confirmed by Folstein et al. with a $100 \%$ sensitivity and specificity (Burns A, 1999).

\section{Data collection}

The patients' spiritual health was assessed using Palutzian \& Ellison's questionnaire, which contains a total of 20 items on religious health (10 odd-numbered items) and existential health (10 even-numbered items). The scores obtained for each of the two dimensions make up the collective score of spiritual health, which ranges from 20 to 120 . Responses are scored based on a six-point Likert scale, ranging from totally disagree (1 point) to totally agree ( 6 points). Negative questions are reverse scored. Spiritual health is ultimately divided into three levels: poor health (20-40), moderate health (4199) and good health (100-120) (Paloutzian et al., 2005). Following translation into Persian, the content validity of the questionnaire was used to ensure its validity and the Cronbach's alpha $(\alpha=0.82)$ to ensure its reliability (Allahbakhshian et al., 2010).

The patients' quality of life was assessed using the standard EORTC QLQ-C30.V3, developed by the European Organization for Research and Treatment of Cancer (Aaronson et al., 1993), which consists of 30 items in five functional domains, including physical (5 items), role functioning ( 2 items), emotional (4 items), cognitive ( 2 items) and social ( 2 items) and nine symptom domains, including the three multi-item domains of fatigue ( 3 items), nausea and vomiting ( 2 items) and pain ( 2 items), and the six single-item domains of shortness of breath, sleep disorder, loss of appetite, constipation, diarrhea and financial problems, and at last a general quality of life domain (2 items) (Gundy et al., 2012). The two items on the general quality of life (items $29 \& 30$ ) were scored based on a Likert scale from very bad (1 point) to excellent ( 7 points) and the remaining items from not at all (1 point) to very much (4 points). The score for each domain ranged from 0 to 100 points (Natrah et al., 2012). In the functional and general quality of life domains, higher scores indicated a better functioning or better quality of life. In the symptoms domains, higher scores indicated the greater severity of that symptom or problem (Bordonaro et al., 2014). The European Organization for Research and Treatment of Cancer has confirmed the validity and reliability of this questionnaire and Montazeri et al. then reported a validity of $76 \%$ and a reliability of $93 \%$ for it in Iran (Montazeri et al., 1999).

Statistical analysis

Following the completion of the questionnaires, 
the collection of the data and their input into SPSS-16, descriptive statistics were used to determine the mean and standard deviation and the tables of frequency distribution and frequency percentage. Inferential statistics were used to determine the relationship between spiritual health and quality of life through the stepwise linear regression analysis. In line with the study objectives and to determine the relationship between spiritual health and quality of life in patients with cancer, thestepwise linear regression

Table 1. Demographic and Clinical Details of Patients with Cancer

\begin{tabular}{|c|c|c|}
\hline Variables/Criteria & Mean & Standard Deviation \\
\hline Age & 50.69 & 13.49 \\
\hline Karnofsky performance status & 71.09 & 12.3 \\
\hline Cognitive status & 25.98 & 1.79 \\
\hline Variables & ntity & Percentage \\
\hline \multicolumn{3}{|l|}{ Gender } \\
\hline Female & 159 & 75.7 \\
\hline Male & 51 & 24.3 \\
\hline \multicolumn{3}{|l|}{ Education } \\
\hline Illiterate & 46 & 21.9 \\
\hline Primary school & 34 & 16.2 \\
\hline Junior high school & 42 & 20 \\
\hline High school & 5 & 2.4 \\
\hline High School Diploma & 45 & 21.4 \\
\hline Associate degree & 10 & 4.8 \\
\hline Bachelor's & 20 & 9.5 \\
\hline Master's & 7 & 3.3 \\
\hline $\mathrm{PhD}$ & 1 & 0.5 \\
\hline \multicolumn{3}{|l|}{ Marital Status } \\
\hline Single & 17 & 8.1 \\
\hline Married & 171 & 81.4 \\
\hline Widowed & 18 & 8.6 \\
\hline Divorced & 3 & 1.4 \\
\hline Separated & 1 & 0.5 \\
\hline \multicolumn{3}{|l|}{ Economic Status } \\
\hline Well-off & 14 & 6.7 \\
\hline Average & 130 & 61.9 \\
\hline Poor & 66 & 31.4 \\
\hline \multicolumn{3}{|l|}{ Type of Cancer } \\
\hline Breast & 107 & 51 \\
\hline Stomach & 15 & 7.1 \\
\hline Colon & 14 & 6.7 \\
\hline Brain & 11 & 5.2 \\
\hline Esophagus & 9 & 4.3 \\
\hline Throat & 8 & 3.8 \\
\hline Oral & 6 & 2.9 \\
\hline Others & 40 & 19 \\
\hline
\end{tabular}

analysis was used. Before performing the analysis, the univariate linear regression analysis was used to identify the confounding factors of the independent and dependent variables, and the variables that were significantly related to the dependent variable $(\mathrm{P}<0.2)$, including age, gender, level of education, Karnofsky status and cognitive status, were added to the main model (the stepwise model) of the test.

\section{Results}

A total of 210 patients with cancer in the age range of 20 to 83 and with a mean age of 50.69 were examined in the study. The majority of the patients were female $(75.7 \%)$ and married $(81.4 \%)$, had breast cancer $(51 \%)$ and rated their own economic status as average (61.9\%). Table 1 presents the demographic and clinical details of the patients examined.

The mean spiritual health score was calculated by adding the mean religious health and existential health scores and was calculated as 78.4 with a standard deviation of 16.12. The mean quality of life score was

Table 2. Mean Scores and Standard Deviation of the Spiritual Health and Quality of Life Variables in Patients with Cancer

\begin{tabular}{lcc}
\hline Variables/Criteria & Mean & Standard Deviation \\
\hline Spiritual health & 78.4 & 16.12 \\
Religious health & 42.68 & 8.1 \\
Existential health & 35.72 & 8.61 \\
Functional status of the quality of life & \\
Physical function & 74.79 & 17.48 \\
Role functioning & 67.93 & 20.77 \\
Emotional function & 45.47 & 22.91 \\
Cognitive function & 80.47 & 24.01 \\
Social function & 73.44 & 23.29 \\
Quality of life signs and symptoms & \\
Fatigue & 49.04 & 20.11 \\
Nausea and vomiting & 11.26 & 18.58 \\
Pain & 38.65 & 23.38 \\
Shortness of breath & 29.12 & 18.43 \\
Sleep disorder & 22.61 & 19.01 \\
Loss of appetite & 33.49 & 28.16 \\
Constipation & 12.18 & 14.24 \\
Diarrhea & 26.95 & 19.36 \\
Financial effects & 54.76 & 34.67 \\
General quality of life & 58.09 & 18.74 \\
\hline
\end{tabular}

Table 3. Stepwise Linear Regression Model for Quality of Life (as a dependent variable) and Spiritual Health (religious and existential dimensions) and Demographic and Clinical Details(as Independent Variables

\begin{tabular}{lcccc}
\hline & $\begin{array}{c}\text { Non-standardized } \beta \\
\text { coefficient }\end{array}$ & $\begin{array}{c}\text { Standardized } \beta \\
\text { coefficient }\end{array}$ & Confidence interval \\
\cline { 3 - 5 } Constant & -75.356 & - & -125.556 & Higher Bound \\
Age & 0.203 & 0.148 & 0.004 & -25.156 \\
Gender & 1.714 & 0.04 & -3.091 & 0.402 \\
Level of education & -0.534 & -0.062 & -1.797 & 6.519 \\
Karnofskyperformance status & 0.917 & 0.606 & 0.713 & -0.73 \\
Cognitive status & 2.205 & 0.214 & 0.295 & 4.12 \\
Spiritual health & 0.795 & 0.688 & 0.708 & 0.115 \\
Religious health & 1.428 & 0.621 & 1.246 & 1.611 \\
Existential health & 1.482 & 0.685 & 1.311 & 1.654 \\
\hline
\end{tabular}


calculated as 58.09 with a standard deviation of 18.74. Of the five functional domains of the quality of life, the weakest was the emotional function $(45.47 \pm 22.91)$ and the strongest the cognitive function $(80.47 \pm 24.01)$. In the nine symptomdomains, the most frequent complaint was about the economic problems ensuing from the disease and the least about nausea and vomiting. Table 2 presents the descriptive details of the sub-groups of spiritual health and quality of life in the patients.

As shown in table 3 , there is a positive $(B=0.688)$ and significant $(\mathrm{r}=0.00)$ relationship between the overall spiritual health score and quality of life in patients with cancer. In other words, patients with a higher spiritual health score enjoy a better quality of life. There were also positive $(\mathrm{B}=0.621$ and $(\mathrm{B}=0.685)$ and significant $(\mathrm{r}=0.00)$ relationships between the religious and existential dimensions of spiritual health and quality of life in patients with cancer.

\section{Discussion}

The results obtained revealed moderate levels of spiritual health and quality of life in the patients studied. Moreover, a positive and significant relationship was observed between spiritual health and quality of life in these patients. The patients also received a higher score in the religious dimension of spiritual health than in the existential dimension, which may be attributed to the dominant cultural and religious background in Iran, as Iranians are generally religious and tend to turn toward religion in the face of crises and stress. This finding concurs with the results obtained in studies conducted by Musarezaie (Musarezaie et al., 2012), Jahani (Jahani et al., 2012) and Ramezankhani (Ramezankhani et al., 2014); however, theydisagree with the results of studies conducted by Allahbakhshian (Allahbakhshian et al., 2010) and Taheri Kharame (Kharame et al., 2013), which showed the higher status of the existential dimension compared to the religious dimension of spiritual health in patients with MS and those undergoing hemodialysis. Thesmallest problem observed in the present study in the dimensions of the quality of life pertained to cognitive functioning, which is confirmed in studies by Kobayashi et al. (Kobayashi et al., 2008) and Blazeby et al. (Blazeby et al., 2003), but is inconsistent with studies by Arndt (Arndt et al., 2004), and Abu-Helalah (Abu-Helalah et al., 2014), which found the smallest problem to pertain to the physical and emotional functions. The present study found the biggest problem or the domain with the lowest score to be emotional function, which is consistent with studies by Pakpour-HAA (Pakpour et al., 2009) and Jafari (Jafari et al., 2013), but inconsistent with the study conducted by Kobayeshi et al. on the effects of socio-economic factors and cancer survivors' concerns on their quality of life (Kobayashi et al., 2008) and another study by Blazeby et al. on the quality of life in patients with cancer (Blazeby et al., 2003), which found the lowest score to pertain to the general quality of life dimension. In the present study, of the nine symptom domains, the most frequent complaint made by the patients was concerned with the economic problems caused by the disease, which is also consistent with the results of studies by Abu-Helalah (Abu-Helalah et al., 2014), Pakpour-HAA (Pakpour et al., 2009) and Jafari (Jafari et al., 2013).

The positive $(\mathrm{B}=0.688)$ and significant relationship found in this study between spiritual health and quality of life in patients with cancer has been previously demonstrated in several studies in relation to various other diseases. A study by Ramezankhani showed statistically significant relationships between spiritual health and the four dimensions of quality of life, including the physical, mental, social and environmental dimensions $(\mathrm{P}<0.05)$ in patients with type II diabetes. In their study, Jafari et al. found a positive and significant correlation between the general quality of life in patients with breast cancer undergoing radiotherapy and their overall score of spiritual health [34]. In another study, Whitford et al. showed a positive and significant relationship between spiritual health and the health-related aspects of the quality of life inAustralian patients with cancer $(r=0.59)$ (Whitford et al., 2008). A study by Baljani showed a positive and significant relationship between spiritual health and the functional scale ofquality of life $(\mathrm{r}=0.23$ and $\mathrm{P}=0.01)$ and also between spiritual health and the overall score of quality of life $(r=0.34$ and $\mathrm{P}<0.01)$ in patients with cancer (Baljani et al., 2011). In a study on spirituality andquality of life in patients with renal failure, Finkelstein demonstrated a direct and significant relationship between spiritual health and the quality of life (Finkelstein et al., 2007). In another study, Allahbakhshian found a significant relationship between spiritual health and the psychological dimension ofquality of life in patients with MS, but no significant relationships between spiritual health and the physical dimension ofquality of life (Allahbakhshian et al., 2010). Jahani et al. also found a significant relationship between spiritual health andquality of life ( $\mathrm{r}=0.94$ and $\mathrm{P}=0.001)$ in their study on patients with coronary artery disease (Jahani et al., 2012). Bekelman also found a statistically significant relationship between spirituality and spiritual health $(\mathrm{r}=0.41$ and $\mathrm{P}=0.001)$ in patients with myocardial infarction (Bekelman et al., 2007). In another study, Kandasamy showed a positive correlation between spiritual health and all the dimensions of quality of life in patients with advanced cancer (Kandasamy et al., 2011). Lucchetti et al. studied patients undergoing hemodialysis and associated high levels of spirituality and religiosity with a better quality of life, a higher system of social support and a greater satisfaction with life (Lucchetti et al., 2010). O'Connor demonstrated a positive and significant relationship between spiritual health and quality of life in patients with blood cancer and revealed patients with higher levels of spiritual health to have received higherquality of life scores (O'Connor et al., 2007). The consistency between the results obtained in the present and the cited studies indicating a significant relationship between spiritual health and quality of life in various patients suggests that quality of life in cancer patients is associated with the faith in God and connection tothe infinite source of power and having a sense of purpose in life. The results of the present study demonstrated a profound relationship between spiritual health and quality of life in patients with life-threatening diseases such as 
cancer, and showed thatpatients whoacquire meaning and purpose in life tend to have a more optimistic view of their disease and are certain about being able to improve their lives, whichcontribute to the improvements made in their quality of life. Devising plansfor improving spiritual health in patients with cancer and presenting strategies for providing spiritual health services can help improve quality of life in these patients. The data presented in the present study were obtained from just one hospital of Tehran and have a reducedgeneralizability of results;however,attempts were made to select the samples from among patients with differing types of cancer in order to increase the applicability of the results, which is a point of strength for the study in comparison with similar studies that haveexamined only one type of cancer.

In conclusion, the significant relationship found between spiritual health and quality of life is further evidencethat the medical communityshould focus on the fourth dimension of health in providing careto cancer patients. Authorities are recommended to provide more comprehensiveand appropriate care services to cancer patients and to dedicate programs and approaches to enhancing their spiritual health. Considering the depth to which spiritual beliefs have penetrated the Iranian society, further studies are recommended to design and implement spiritually-based interventions and methodsof providing spiritual services aimed at improving quality of life in patients with cancer and to also assess the relationship between spiritual health and the degree of progress and the improvementsmade in the quality of life of patients with cancer.

\section{Acknowledgements}

The authors would like to express their gratitude to all the officials at the Cancer Institute of Iran for their cooperation. This studyis part of the results ofamaster's thesis approved and funded by Qazvin University of Medical Sciences.

\section{References}

Aaronson NK, Ahmedzai S, Bergman B, et al (1993). The European Organization for Research and Treatment of Cancer QLQ-C30: a quality-of-life instrument for use in international clinical trials in oncology. $J$ Nat Cancer Institute, 85, 365-76.

Abu-Helalah MA, Alshraideh HA, Al-Hanaqta MM, et al (2014). Quality of life and psychological well-being of colorectal cancer survivors in Jordan. Asian Pac J Cancer Prev, 15, 7653-64.

Akyuz A, Guvenc G, Ustunsoz A, et al (2008). Living with gynecologic cancer: experience of women and their partners. J Nurs Scholarsh, 40, 241-7.

Allahbakhshian M, Jaffarpour M, Parvizy S, et al (2010). A Survey on relationship between spiritual wellbeing and quality of life in multiple sclerosis patients. Zahedan J Res Med Sci, 12, 29-33.

Arndt V, Merx H, Stegmaier C, et al (2004). Quality of life in patients with colorectal cancer 1 year after diagnosis compared with the general population: a population-based study. J Clin Oncol, 22, 4829-36.

Baider L, Ever-Hadani P, Goldzweig G, et al (2003). Is perceived family support a relevant variable in psychological distress?. A sample of prostate and breast cancer couples.J Psychosom Res, 55, 453-60.

Baljani E, Kazemi M, Amanpour E, et al (2011). A survey on relationship between religion, spiritual wellbeing, hope and quality of life in patients with cancer. $J$ Nurs Midwifery, Mashhad, 1, 51-62.

Bekelman DB, Dy SM, Becker DM, et al (2007). Spiritual wellbeing and depression in patients with heart failure.J General Internal Med, 22, 470-7.

Blazeby J, Conroy T, Hammerlid E, et al (2003). Clinical and psychometric validation of an EORTC questionnaire module, the EORTC QLQ-OES18, to assess quality of life in patients with oesophageal cancer. European Journal of Cancer, 39, 1384-94.

Bordonaro S, Vizzini L, Spinnato F, et al (2014). Oral chemotherapy in elderly patients with advanced non small cell lung carcinoma. WCRJ, 1, 223.

Bottomley A (2002). The cancer patient and quality of life. Oncologist, 7, 120-5.

Burns A LB, Craig S 1999. Assessment scales in old age psychiatry, Martin Dunitz.

Finkelstein FO, West W, Gobin J, et al (2007). Spirituality, quality of life and the dialysis patient. Nephrology Dialysis Transplantation, 22, 2432-4.

Gundy CM, Fayers PM, Groenvold M, et al (2012). Comparing higher order models for the EORTC QLQ-C30. Quality of Life Research, 21, 1607-17.

Jafari N, Farajzadegan Z, Zamani A, et al (2013). Spiritual well-being and quality of life in Iranian women with breast cancer undergoing radiation therapy. Support Care Cancer, 21, 1219-25.

Jahani A, Rejeh N, Heravi-Karimooi M, et al (2012). The Relationship Between Spiritual Health and Quality of Life in Patients with Coronary Artery Disease. Islamic Lifestyle Centered on Health, 1, 19-24.

Kandasamy A, Chaturvedi SK, Desai G (2011). Spirituality, distress, depression, anxiety, and quality of life in patients with advanced cancer. Indian J Cancer, 48, 55-9.

Kobayashi K, Morita S, Shimonagayoshi M, et al (2008). Effects of socioeconomic factors and cancer survivors' worries on their quality of life (QOL) in Japan. Psychooncology, 17, 606-11.

Lucchetti G, Almeida LG, Granero AL (2010). [Spirituality for dialysis patients: should the nephrologist address?]. J Bras Nefrol, 32, 126-30.

Manandhar S, Shrestha DS, Taechaboonsermsk P, et al (2014). Quality of life among breast cancer patients undergoing treatment in national cancer centers in Nepal. Asian Pac J Cancer Prev, 15, 9753-7.

Mauk KL, Schmidt NA 2004. Spiritual care in nursing practice, Lippincott Williams \& Wilkins.

McClain CS, Rosenfeld B, Breitbart W (2003). Effect of spiritual well-being on end-of-life despair in terminally-ill cancer patients. The Lancet, 361, 1603-7.

Montazeri A, Harirchi I, Vahdani M, et al (1999). The european organization for research and treatment of cancer quality of life questionnaire (EORTC QLQ-C30): translation and validation study of the Iranian version. Supportive Care in Cancer, 7, 400-6.

Mousavi SM, Gouya MM, Ramazani R, et al (2009). Cancer incidence and mortality in Iran. Ann Oncol, 20, 556-63.

Musarezaie A, Naji Esfahani H, Momeni GHaleghasemi T, et al (2012). The relationship between spiritual wellbeing and stress, anxiety, and depression in patients with breast cancer. Isfahan Medical School, 30.

Natrah M, Ezat SW, Syed M, et al (2012). Quality of life in Malaysian colorectal cancer patients: a preliminary result. 
Rafat Mohebbifar et al

Asian Pac J Cancer Prev, 13, 957-62.

O'Connor M, Guilfoyle A, Breen L, et al (2007). Mental health, religion and culture, 10, 631-47.

Pakpour HAA, Panahi CD, Yekaninezhad S, et al (2009). Assessment of quality of life in patient with esophagus cancer. J Iranian Institute Health Sci Res, 8, 371-8.

Paloutzian RF, Park CL, Paloutzian R, et al (2005). Integrative themes in the current science of the psychology of religion. Handbook of the psychology of religion and spirituality, 3-20.

Polonsky WH (2000). Understanding and assessing diabetesspecific quality of life. Diabetes spectrum, 13, 36 .

Ramezankhani A, Ghaedi M, Hatami H, et al (2014). Association between spiritual health and quality of life in patients with type 2 diabetes in Bandar Abbas, Iran. Bimonthly Journal of Hormozgan University of Medical Sciences, 18, 229-37.

Reb AM (2007). Transforming the death sentence: elements of hope in women with advanced ovarian cancer. Oncol Nurs Forum, 34, 70-81.

Rezaei M, Seyed Fatemi N, Hoseini F (2008). Spiritual wellbeing in Cancer Patients who undergo chemotherapy. Hayat, 14, 33-9.

Riley BB, Perna R, Tate DG, et al (1998). Types of spiritual well-being among persons with chronic illness: their relation to various forms of quality of life. Arch Phys Med Rehabil, 79, 258-64.

Rose P, Yates P (2001). Quality of life experienced by patients receiving radiation treatment for cancers of the head and neck. Cancer Nurs, 24, 255-63.

Rustøen T, Cooper B, Miaskowski C (2010). The importance of hope as a mediator of psychological distress and life satisfaction in a community sample of cancer patients. Cancer Nurs, 33, 258-67.

Siegel RL, Miller KD, Jemal A (2015). Cancer statistics, 2015. CA: A Cancer J Clin, 65, 5-29.

Taheri Kharame Z, Asayesh H, Zamanian H, et al (2013). Spiritual well-being and religious coping strategies among hemodialysis patients. IJPN, 1, 48-54.

Torre LA, Bray F, Siegel RL, et al (2015). Global cancer statistics, 2012. CA: A Cancer J Clin, 65, 87-108.

Vrinten C, van Jaarsveld CH, Waller J, et al (2014). The structure and demographic correlates of cancer fear. BMC cancer, 14, 597.

Whitford HS, Olver IN, Peterson MJ (2008). Spirituality as a core domain in the assessment of quality of life in oncology. Psycho-Oncology, 17, 1121-8. 